\title{
Theaterarbeit an der Sprache
}

\author{
Zur Archäologie der Subtexte im Fremdsprachenunterricht
}

\section{Uta Schorlemmer}

\begin{abstract}
Zusammenfassung
Die 'archäologische' Arbeit an Subtexten ist eine spielerische Erweiterung von Texten um Spielräume des Ungesagten und der Interpretation. Der Leser 'unterlegt' dem Text seine individuelle Wahrnehmung und körperliche Erfahrung. Übungen zu Subtext und inneren Monologen können ein wirkungsvolles Instrument der Textarbeit im Fremdsprachen-unterricht, aber auch Grundlage szenischen Spiels sein, dessen Impuls nicht psychisch, sondern körperlich ausgelöst wird.
\end{abstract}

\section{Einsatz}

Ziel dieses Beitrags ist es, dem Manko entgegenzutreten, das in den meisten mir bekannten dramapädagogischen Aufsätzen herrscht, nämlich dass nur selten erklärt wird, auf welche Vorbilder im Theater sich die Autoren im Einzelnen beziehen. Mittlerweile gibt es erfreulicherweise eine bemerkenswerte Menge an Literatur zur Dramapädagogik. Meist wird darin auf ein recht allgemein gehaltenes Theaterverständnis zurückgegriffen, das mit dem Sprachlernprozess verbunden wird, oft basierend auf theaterpädagogischen Ansätzen. Die letzteren haben sich ihrerseits ursprünglich an konkret benannten Theaterkonzepten orientiert, die sich durch die theaterpädagogische Praxis aber häufig verselbständigt haben. Das ist nicht unbedingt negativ zu bewerten, denn natürlich weist dieser Verkürzungs- und Fokussierungsvorgang auf die Etablierung einer eigenen wissenschaftlichen Disziplin hin, sowohl jenseits der Philologien als auch der Theaterwissenschaft. Dennoch scheint es mir nicht nur aufschlussreich, sondern auch geboten zu sein, diesem Manko aktiv zu begegnen, indem anhand des Beispiels der Arbeit an Subtexten die zugrunde liegenden Theaterkonzepte unmittelbar auf den dramapädagogischen Sprachlernprozess übertragen werden. Der vorliegende Beitrag ${ }^{1}$ stellt einen Anfang dazu dar, den 'Aufriss' eines Teils eines für 2010 geplanten Forschungsprojektes.

Ähnlich den Methoden der Theater-Aktionsforschung hat Brecht mit der Dokumentation seiner Theaterarbeit (1990) versucht, die Entwicklung szenischer

\footnotetext{
${ }^{1}$ Der Text basiert teilweise auf meinem Vortrag "Spielend lesen - (Kon)Texte schaffen", den ich am 5. Juni 2009 bei der 12.Grazer Tagung DaF/DaZ gehalten habe.
} 
Vorgänge möglichst genau und nachvollziehbar zu dokumentieren. Dies soll für das anstehende Forschungsprojekt als methodische Anregung herangezogen werden. Die praktischen Beispiele im vorliegenden Beitrag beziehen sich allerdings nur zum Teil auf bereits im Unterricht ausprobierte Übungen.

Ich will mich hier auf den Begriff des Subtextes und dessen Gebrauch im Fremdsprachenunterricht konzentrieren. Den Begriff leite ich aus dem System des späten Konstantin Stanislavski ab und verbinde ihn mit dem konstruktivistischen Theater Vsevolod Meyerholds sowie mit Bertolt Brechts Konzept des Verfremdungseffekts. Arbeit mit dem Subtext bedeutet spielerische Erweiterung des Ausgangstexts um Spielräume des nicht explizit Ausgedrückten und der individuellen Interpretation. Es handelt sich um eine Art 'Textarchäologie'. Wolfgang Iser spricht vom Akt des Lesens als Konstitution von Imaginationen (cf. 1984). Der „literarische Sprechakt" sei ein Appell zur „Leerstellenergänzung“ (1984: 284). Wie in Umberto Ecos Konzept des Lector in fabula (Eco 1994) erschafft der Leser den Text beim Lesen mit, indem er die Worte des Autors vor dem Hintergrund des Hier und Jetzt sowie seiner Persönlichkeit, Lebenserfahrung etc. versteht und den Text damit um seine individuelle Wahrnehmung ergänzt. Der polnische Regisseur Krystian Lupa, dessen Probenarbeit vorwiegend aus der intensiven Arbeit an Subtexten besteht, spricht von 'Palimpsestes', die einen Text ausmachen und die im Prozess der Inszenierung freizulegen sind. Den Begriff entlehnt er dem Griechischen: Im Altertum bezeichnete man mit 'Palimpseste' mehrfach überschriebene Handschriften, bei denen im Verlauf der Zeit die einzelnen Schichten ihrer ehemaligen Beschriftungen nur noch schemenhaft sichtbar waren, aber dabei dennoch das Gesamtschriftbild prägten.

In welchem theatralen Kontext ich den Begriff des Subtexts sehe und wie er als Instrument im Fremdsprachenunterricht verwendet werden kann, will ich in den nächsten beiden Abschnitten zunächst theoretisch als Spielanleitung erläutern, um dies im Anschluss in einem Spiel, d.h. einem Unterrichtsdesign, ausführen.

\section{Spielanleitung}

\subsection{Der Begriff des Subtextes und seine Fundierung im Theater}

Stanislavski hat in dramatischen Texten eine Ebene des nicht Ausgesprochenen entdeckt, die er "Unterwasserstrom" (Stanislavski, zitiert in Schmid 1992: 65) nannte. Dafür hat sich der Terminus Subtext durchgesetzt. Während der Proben erschließt sich der (lesende) Schauspieler beim Zwischen-den-Zeilen-Lesen den Inhalt des Textes entsprechend seiner Kenntnis des Kontextes und der Situation sowie in Konfrontation mit der eigenen Person. Was der russische Schauspieltheoretiker zunächst als psychisch dominiertes Einfühlen in die Figur entwickelte, modifizierte er innerhalb seines Systems zur Methode der physischen Handlungen, bei der der Körperimpuls primär ist.

Meine Methode beruht darauf, die inneren und äußeren Vorgänge miteinander zu verbinden und das Gefühl für die Rolle durch das physische 
Stanislavski ist über viele Jahre als Regisseur und Theoretiker des naturalistischen Theaters rezipiert worden, was vor allem mit der schwierigen Quellenlage, aber auch mit tendenziösen amerikanischen Übersetzungen sowie der Weiterentwicklung seines Systems durch Theaterleute wie Lee Strassberg zustande kam.

[Stanislavkis] Regiebücher vermitteln nämlich nicht das Bild eines Künstlers, der auf die psychischen Motivationen des Schauspielers fixiert ist, sondern das Bild eines Regisseurs, der von außen auf das Geschehen blickt und bereits mit antinaturalistischen Techniken experimentiert. (Ahrends 1992: 9)

Weit über das pure Auswendiglernen hinaus muss der Text im Theater (egal aus welcher Quelle er stammt, d.h. ob er improvisiert oder vorgegeben wird), vom Sprecher angenommen werden. Ich vermeide bewusst Worte wie 'verinnerlicht' oder 'einverleibt', denn was ich hier hervorheben möchte, ist nicht in erster Linie die passive innerliche, emotionale Reaktion auf den Text, sondern die körperliche und kommunikative Aktion des Sprechers. Somit führt der Subtext zum Begriff der Verkörperung oder englisch embodiment, d.h. der Text wird durch den Körper und dessen gestisches, mimisches, kinetisches und stimmliches Potential kommuniziert.

Viel prononcierter und in anderer Weise als Stanislavski setzt dessen (abtrünniger) Schüler Vsevolod Meyerhold auf den körperlichen Einsatz als Ausgangspunkt szenischer Übungen. Sein historischer Bezugspunkt ist der Balagan, eine Jahrmarktstheaterbude, wo insbesondere die Poesie des Puppenspiels von der imperfekten Körperlichkeit der Marionetten lebt. Auch in Meyerholds konstruktivistischem Ansatz passiert im Kopf des Zuschauers eine „Leerstellenergänzung“ (cf. Iser).

In der Kunst haben wir es immer mit der Organisation des Materials zu tun. Der Konstruktivismus forderte vom Künstler, dass er auch Ingenieur sei. Die Kunst sollte auf wissenschaftlichen Grundlagen basieren, das ganze Schaffen des Künstlers soll bewusst sein. Die Kunst des Schauspielers besteht in der Organisation seines Materials, das heißt im Vermögen, die Ausdrucksmittel seines Körpers richtig anzuwenden. Im Schauspieler vereinen sich sowohl Organisator als auch Organisierter (also Künstler und Material). In einer Formel für Schauspieler fände das folgenden Ausdruck:

$\mathrm{N}=\mathrm{A} 1+\mathrm{A} 2$

wobei $\mathrm{N}=$ Schauspieler

A1 = Konstrukteur, der plant und Anordnung für die Realisierung der Ideen gibt, A2 = Körper des Schauspielers, der die Aufgaben des Konstrukteurs (A1) ausführt.

(Meyerhold 1967a: 101f) 
Meyerhold geht so weit zu behaupten, dass für ihn die Worte im Theater „nur Muster im Gewebe der Bewegungen“ (Meyerhold 1967b: 71) darstellten und betont, dass die „richtige Schauspielmethode [...] nicht vom Inneren zum Äußeren, sondern umgekehrt: vom Äußeren zum Inneren" (Meyerhold 1967a: 103) zu entwickeln sei. Das wichtigste historische Verdienst von Theaterrevolutionären wie Meyerhold war die Reetablierung des Prinzips der Maske und also eines anti-illusionistischen Theaters, in dem die vierte Wand eingerissen wurde. ${ }^{2}$ Das Geschehen findet im Theater Meyerholds wie auch beispielsweise Tadeusz Kantors auf der Bühne statt, die sich nicht durch Einfühlung in die imaginierte Welt verwandelt, sondern auf der ein Spiel der maskierten Körper mit der Imagination eines jeden Anwesenden stattfindet. Dort, in der Phantasie, entwickelt sich, blüht und vergeht die Welt der Dichtung. Die Bühne ist ein Ort des Konjunktivs, dort könnte etwas geschehen, aber es ist fiktiv, an die Gesetze der Bühne gebunden und vom Schauspieler bewusst für den vergänglichen Moment erschaffen. Brecht spitzt dies für sein Theater durch die Haltung des Zeigens noch zu: Der Schauspieler versetzt seinen Körper mit seiner von der Gegenwart her geprägten Phantasie in die Lage des Fremden und führt diesen Vorgang dem explizit aktiven Zuschauer vor, so dass dieser sich selbst ein Bild machen kann.

Die genannten Beispiele aus der Schauspielmethodik treffen sich mit Erkenntnissen der Entwicklungspsychologie, die in der Geste den Ursprung menschlicher Kommunikation erkennt (cf. Jérôme Bruner 1961, zitiert in Wagner 1998: 21). Die drei Ebenen, mit denen ein Individuum mit Realität umgeht, sind „enaktiv“, „ikonisch“ und „symbolisch“. Bruner geht in seiner entwicklungspsychologischen Theorie davon aus, dass in kindlichen Lernprozessen diese drei Repräsentationsmodi zugleich aktiviert werden. Auch der russische Entwicklungspsychologe Lev Vygotsky geht davon aus, dass Sprechen ursprünglich und wesentlich eine soziale Funktion hat (cf. Vygotsky 2002: 94). Dies ist bereits vielfach auf den Sprachlernprozess übertragen worden. Auch der Lernerfolg kinästhetischer Lernstrategien konnte bereits vielfach nachgewiesen werden (cf. Wagner 1998).

Vygotsky hat parallel zu Stanislavski den Begriff des „inneren Sprechens“ (2002: 44) entwickelt, den er zwischen den Gedanken und dem Sprechen verortet. $^{3}$ Der Übergang vom inneren zum äußeren Sprechen sei jedoch keine einfache Addition der Lautseite zur stummen Sprache, keine direkte Übersetzung, sondern eine Transformation,

[...] eine Umstrukturierung des Sprechens, eine Umwandlung der völlig urwüchsigen und eigenartigen Syntax sowie der semantischen und lautlichen Struktur des inneren Sprechens in andere Strukturformen, die dem äußeren Sprechen eigen sind. Genauso wie inneres Sprechen nicht Sprechen minus Laut ist, so ist äußeres Sprechen nicht inneres Sprechen plus Laut. [...] Inneres Sprechen erweist sich als dynamisches, instabi-

\footnotetext{
${ }^{2}$ Auch Stanislavski entfernte sich im Laufe seines Schaffens von der Idee der absoluten vierten Wand. Und damit der perfekten Illusion im Theater.

${ }^{3}$ Stanislavski und Vygotsky waren gut miteinander bekannt.
} 
les Moment, das zwischen Wort und Gedanke als den ausgeformteren und stabileren extremen Polen des verbalen Denkens oszilliert. (Vygotsky 2002: 456f)

Denken ist laut Vygotsky nicht vom Affekt zu trennen (cf. Vygotsky 2002: 54f). In der Hirnforschung (cf. Damásios 1994, Goleman 1995) wird der Nachweis erbracht, dass die gleichen zerebralen Strukturen sowohl für emotionale als auch kognitive Prozesse verantwortlich sind, d.h. Kognition und Emotion lassen sich nicht voneinander trennen. Emotionen sind zudem von vielen inneren und äußeren Faktoren abhängig und u.a. situationsbedingt. Relevanz im theatralen Prozess erlangen sie erst dann, wenn sie mitteilbar sind. Wenn wir hier die Körperlichkeit szenischer Situationen zu erfassen versuchen (ohne dabei Gefühle ausschalten zu wollen), so liegt der Fokus dabei also nicht auf dem individuellen Erleben-für-sich-selbst, wie Stanislavski es in seinen frühen Schriften zur Arbeit des Schauspielers an sich selbst beschreibt, sondern um die $\ddot{A} u ß e r u n g$ von körperlichen und emotionalen Zuständen des spielenden Menschen.

In diesem Zusammenhang ist eine Feststellung Vygotskys über die Beziehung zwischen Denken und Sprechen interessant:

Das Sprechen ist seiner Struktur nach keine spiegelhafte Abbildung der Struktur des Denkens. Es kann deshalb dem Denken nicht wie ein fertiges Kleid übergestülpt werden. Das Sprechen dient nicht als Ausdruck des fertigen Gedankens. Indem sich der Gedanke in Sprechen verwandelt, gestaltet er sich um, verändert sich. Der Gedanke drückt sich im Wort nicht aus, sondern vollzieht sich im Wort. (Vygotsky 2002: 401)

Daraus lässt sich die Beobachtung ableiten, dass die innere Erlebenswelt eines Individuums immer nur in vermittelter Form weitergegeben werden kann, d.h. sie muss übersetzt werden in andere, auf Kommunikation ausgerichtete Ausdrucksformen. Die Arbeit an Subtexten entspricht einer solchen Transformation von Gedanken in Sprechen, wobei dieser Prozess laut Vygotsky ein dialektischer ist.

Wie sich diese theoretischen Vorgaben in meinem Ansatz für dramapädagogischen Unterricht widerspiegeln, ist Anliegen des nächsten Abschnitts.

\section{2 Überlegungen zu Subtexten und physischen Aktionen im Sprachunterricht}

Dorothy Heathcote meint zu Recht, „das Hauptziel von Dramapädagogik besteh[e] darin, die Schüler dazu anzuhalten, hinter die sich auf der Oberfläche abzeichnende Handlung zu schauen." (Heathcote \& Bolton 1998: 160, Übers. US) Sie stelltheraus, dass sich Kommunikation sowohl aus dem Gehörten als auch dem nicht Gehörten, dem Gesehenen als auch dem nicht Gesehenen entwickelt. Dass dabei der körperliche Impuls den Spieler leitet, ist, meine ich, unter den gegebenen Umständen des Sprachunterrichts (zeitliche Beschränkung, kaum 
Theatererfahrung der Teilnehmer etc.) eine viel fruchtbarere Grundannahme als zu sehr mit sogenannten 'echten' Gefühlen zu spielen.

Werden Texte im Unterricht nach dem Modell des Subtextes interpretiert, so liegt das Hauptaugenmerk hier nicht auf dem Abfragen eines in der Fremdsprache erfassten Informationsgehaltes, der sich häufig genug bei geschickten Lernern auf Wiederholung bzw. Wiedererkennung sprachlicher Muster beschränkt, sondern auf einer offenen Textinterpretation, die sowohl verschiedene Schichten (s. Palimpsestes) insbesondere von literarischen Texten freilegt als auch das Hier und Jetzt der Textrezeption berücksichtigt. Es gibt kein Rezept für die spielerische Interpretation eines Textes, sondern jede neue Bearbeitung im Sinne von Subtextualisierung und dem darauf aufbauenden szenischen Spiel mit physischen Aktionen ist kontextabhängig. In dieser Einschränkung auf einen spezifischen Kontext liegt aber die große Freiheit jedes Spiels. Meyerhold wirft jedoch zu Recht das Problem der Technik des Körperspiels auf, das im Sprachunterricht eine besondere Facette hat, weil das Ziel sich von der Theateraufführung auf den Sprechakt verschiebt. In Meyerholds Methode der Biomechanik, in der er innerhalb seiner eigenen Schauspielschule ein ganzes Repertoire an physischen Aktionen in Etüden und Übungsabläufen entwickelt hat, sehe ich eine Fundgrube für die Dramapädagogik. Dieser Spur werde ich im erwähnten Forschungsprojekt weiter nachgehen.

Vygotskys Begriff des „inneren Sprechens“ ist für den Sprachunterricht in zweierlei Hinsicht relevant. Zum einen greift er die Sprechmotivation auf. In unserem Zusammenhang bietet die Aufgabe, einen Subtext zu entwickeln, die Basis zur Kommunikation. Dabei ist es wichtig, sich über die Beziehung zwischen Denken und Sprechen bewusst zu sein, um in der dramapädagogischen Arbeit effektiv damit umzugehen. Eine alltägliche Sprechsituation beginnt, meist eher unbewusst, mit „innerem Sprechen“. Beim Gebrauch einer Fremdsprache hingegen, in der aufgrund fehlender Erfahrungen je nach Sprachniveau seltener automatisierte Sprechhandlungen möglich sind, lässt sich der Prozess des 'Reifens' des passenden sprachlichen Ausdrucks als Aktivierung des Gelernten bzw. vielmehr als kreative Kombination von Bruchstücken angeeigneter Sprachmuster beschreiben. Vygotskys Theorie ist auch deshalb für den dramapädagogischen Unterricht interessant, weil hier das Sprechen bei Kindern über kinästhetisch-motorische Übungen gefördert werden soll, was wiederum mit dem hier vorgestellten Theateransatz der physischen Aktionen korreliert. Zudem handelt es sich beim Sprechvorgang selbst ebenfalls um einen physischen Akt.

Dramapädagogischer Unterricht ist generell geeignet, Sprechhemmungen abzubauen. Das funktioniert meiner Ansicht nach besser, wenn nicht psychologische, sondern physische Komponenten den Prozess des Sprechens in Gang setzen. Aufgrund bestimmter Themen bzw. dem zufälligen Verlauf einer Improvisation in Kombination mit der persönlichen Situation einzelner Teilnehmer kann es im dramapädagogischen Unterricht zu Situationen kommen, die einzelne Teilnehmer persönlich über Gebühr emotional berühren. Vor dieser Gefahr hat unter anderem Even gewarnt, bezogen auf szenisches Arbeiten, das 
'too close to home' sein könnte (cf. Even 2003: 169). Der hier dargelegte Ansatz, der Erfahrung über Einfühlung stellt, kann hilfreich sein bei der Vermeidung von Situationen im Unterricht, die der Psychotherapie nahe kommen. Auf der Ebene der Textinterpretation geht damit die Methode der Verfremdung und des Spiels mit assoziierten Begriffen einher (s. 3.1. Vor Spielbeginn).

Die Konzepte des bewussten, nicht illusionistischen Spiels sowie einer Zuschaukunst im Sinne Brechts, bei der der Zuschauer ebenso aktiv und kreativ ist wie der Schauspieler, sind für den dramapädagogischen Unterricht, wieich ihn verstehe, zentral. Auch der Brechtsche Gedanke, dass das szenisch Dargestellte selbst veränderbar ist und damit auf die Veränderbarkeit der Gesellschaft hindeutet, scheint mir für das dramapädagogische Arbeiten wichtig zu sein, das meistens als Prozess aufgefasst wird, bei dem jede Unzulänglichkeit produktiv gemacht werden kann.

Mit dem hier formulierten Theaterverständnis und seinem Bezug zur Dramapädagogik sei vor allem eine Herangehensweise skizziert, die das Selbstverständnis der Lehrperson und der Gruppe insgesamt, die Wahl der Mittel, die zurVerfügung gestelltwerden, die Art der Textinterpretation sowie die Spielweise beeinflusst. Dies soll im Folgenden anhand eines Unterrichtsdesigns zum Subtext dargelegt werden, ergänzt durch eine Unterform des Subtextes, den inneren Monolog. Den letzteren Begriff entlehne ich den Proben des polnischen Regisseurs Krystian Lupa, dessen Theaterarbeit ich eine Zeit lang im Rahmen meiner Dissertation begleitet habe. Er ist nicht equivalent zum „inneren Sprechen“, wie Vygotsky es definiert. Innerer Monolog meint in Lupas Theater einen nicht ausgesprochenen Text, der den gesprochenen Zeilen im Probenprozess bewusst und aktiv unterlegt wird, hier auch formuliert, der aber dann in der ausgearbeiteten Szene verinnerlicht ist. Es ist der präsente, aber nicht hörbare Subtext einer Figur während sie auf der Bühne ist. Übertragen auf meine folgenden Erläuterungen zur Verwendung von Subtexten in Sprachlernprozessen, verstehe ich den inneren Monolog als den Subtext einer einzelnen Figur in einer bestimmten Situation im Unterschied zum generellen Subtext der Situation, bei dem nach deren Details zu Ort, Zeit, Beziehungen der Figuren untereinander etc. gefragt wird.

\section{Spiel}

\subsection{Vor Spielbeginn}

Prinzipiell kann ich mir alle möglichen Textsorten für Subtextübungen vorstellen. Ich habe hier einen Prosatext ausgewählt: eine sprachlich vereinfachte und episodisch für ein Bilderbuch erzählte Version des Märchens Jorinde und Joringel der Gebrüder Grimm (Grimm, Steiner 2001), in dem ein Geschwisterpaar in einen verwunschenen Wald gerät, wo das Mädchen von der Zauberin in eine Nachtigall verwandelt wird, wovon ihr Bruder sie mit Hilfe einer Wunderblume schließlich befreit. 
Sowohl für dramatische ${ }^{4}$ Theatertexte als auch für Prosatexte kann zwischen Subtext, der nicht zwingend an eine bestimmte Figur gebunden ist, und innerem Monolog, der das nicht Gesagte aus der Perspektive einer bestimmten Figur verbalisiert, differenziert werden. Den inneren Monolog will ich als personalisierte Form des Subtextes definieren, um die beiden Ebenen klarer zu differenzieren. Subtexte und innere Monologe brauchen nicht unbedingt eine Textvorlage, sondern können auch auf Vorgaben zu szenischen Situationen mit den entsprechenden Körperhaltungen basieren. ${ }^{5}$

Relevante Komponenten für Subtexte und innere Monologe sind:

- Raum, Atmosphäre, Wetter, Licht etc.

- historische Zeit, Jahres- und Tageszeit

- Verhältnis der Figuren untereinander und Kommunikationssituation

- Körperlichkeit und momentaner persönlicher Zustand der Figuren in Bezug auf die szenische Situation

- (fiktive) Biographie der Figuren

Informationen dazu können mit Hilfe von Fragen erarbeitet bzw. erfunden werden. Diese orientieren sich an den 5 W-Fragen des Theaters: Wer? Was? Wann? Wo? Warum?, hinter denen sich weitere Fragen nach dem Wie?, Woher? etc. verbergen. Die Fragen sollten dem Sprachniveau angepasst und je nach Ausgangstext entsprechend der gegebenen Figurenkonstellation, Situation etc. modifiziert werden. Zu manchen Fragen sind konkrete Antworten im Text zu finden. Es gibt aber auch eine ganze Reihe der Phantasie überlassener Begebenheiten. Dabei - wie oben angedeutet - verfremdend vorzugehen, bedeutet konkret, dass die im Text beschriebene Situation (oder die vom Sprachlerner davon nach der ersten Lektüre verstandenen Fragmente) zunächst in ihrem vorgegebenen Kontext und also in ihrer Fremdheit wahrgenommen wird. Erst in einem nächsten Schritt wird dieses explizit Fremde auf die eigene Wirklichkeit bezogen und zwar von einem Lernenden, der „Ingenieur“ seines Handelns ist, wie es bei Meyerhold (1967: 101) heißt . Beim assoziativen Zusammentragen von Wörtern oder Wortgruppen für Subtexte ist es geradezu erwünscht, dass auch auf den ersten Blick zusammenhanglos wirkende Assoziationen aufgenommen werden. Das kann zu abstrakteren Kombinationen führen, die ein hohes dramatisches

\footnotetext{
${ }^{4}$ Ich benutze den Begriff 'dramatisch' im Unterschied zu „postdramatisch“ (cf. Lehmann 1999), wo die Form des Dialogs nicht mehr unbedingt überwiegt.

${ }^{5}$ Das Video eines meiner Studenten im ersten Semester Deutsch am Occidental College in Los Angeles im Herbst 2009 ist ein gutes Beispiel dafür, wie aus Körperhaltungen ein innerer Monolog entstehen kann, der im Film auch zum Teil in Worte gefasst wird. Jeven Doveys Clip Der Raum ist einsehbar unter http://www. youtube.com/watch?v=u3M5HB1ouFo. Selbst an diesem Filmmaterial lässt sich im Unterricht im Sinne weiterer Verbalisierungen des Subtextes beider Figuren arbeiten.
} 
Potential im Sinne apsychologischer, nicht-illusionistischer Darstellung haben und zugleich Vokabular aktivieren, das aus ungewöhnlichen Quellen (zum Beispiel aus klanglichen Assoziationen) herrührt und in anderen, weniger arbiträren, sondern eher linearen Textinterpretationsmethoden nicht zum Zuge käme. Der assoziative Prozess der Entwicklung von Subtexten und inneren Monologen trägt seinerseits zur Verfremdung bei.

In dem folgenden Katalog habe ich mögliche Fragen aufgeführt, aus denen für die konkrete Unterrichtssituation eine Auswahl getroffen werden kann. Sie müssen nie in Reihenfolge beantwortet werden, denn die Prioritäten bzw. Assoziationsspielräume werden von den Texten selbst vorgegeben.

- WER? Welche Figuren gibt es in dem Abschnitt? Von welchen ist außerdem die Rede? Wie alt sind sie? Was ist ihr Beruf? Wie stehen sie zueinander? u.a.

- WO? Wo sind die Figuren? Wie sieht es dort genau aus? Wer befindet sich wo? u.a.

- WANN? In welcher historischen Zeit und zu welcher Jahres-, Tages-, Uhrzeit passiert die Begebenheit? u.a.

- WAS? Was passiert? Was tun die Figuren? In welcher körperlichen Haltung und Verfassung befinden sie sich? Was denken und fühlen sie (innerer Monolog)? u.a.

- WARUM? Warum und wie kommt es zu dem Ereignis? Was sind die Gründe für ein bestimmtes Verhalten? u.a.

In dem hier entworfenen Spiel konzentriere ich mich auf die Entwicklung sprachlicher Erweiterungen des Originaltextes. Ich führe anhand der ersten Abschnitte des Märchens Möglichkeiten auf, wie Subtexte und innere Monologe $\mathrm{zu}$ Jorinde und Joringel entwickelt werden können, die sicherlich modifizierbar sowie dem Sprachniveau als auch der gegebenen Unterrichtszeit anzupassen sind. Im Folgenden gehe ich prinzipiell nach Evens Unterrichtsphasenmodell (Sensibilisierungsphase, Kontextualisierungsphase, Einordnungsphase, Intensivierungsphase, Präsentation und Reflexion) vor, ohne jede einzelne Phase genauer zu beleuchten.

Ich empfehle diese Skizze eines Unterrichtsdesigns für die Niveaustufen A2 bis B2, wobei der Text für niedrigere Niveaus eventuell sprachlich noch weiter vereinfacht werden müsste.

\subsection{Spracharbeit am Subtext eines Märchens}

In der Sensibilisierungsphase der Gesamtgruppe halte ich es für günstig, bereits mit dem ersten Abschnitt des Märchens zu beginnen: 
In dieser Geschichte kommen sieben tausend Vögel, eine Zauberin, ein Liebespaar und eine rote Wunderblume vor, und weil sie einen Anfang haben muss, fängt sie an, wie viele Märchen - mit einem Schloss und mit einem Wald und mit einem Satz: Es war einmal... (Grimm, Steiner 2001)

Dabei sind Aufwärmübungen wie ein mit farbigen Vögeln variiertes ObstsalatSpiel denkbar. Im Ursprungsspiel wird jedem Mitspieler eine Obstsorte zugeteilt, hier variiert mit roten, grünen, gelben Vögeln, die zusammen einen Schwarm ergeben. Die Gruppe setzt sich in einen Stuhlkreis, ein Spieler verbleibt in der Mitte ohne Stuhl. Dieser sucht sich eine oder mehrere vorher festgelegte Vogelsorten aus, die er zusammen 'mixt', d.h. deren Namen er nennt. Die Genannten müssen die Plätze tauschen. Es ist aber jeweils ein Platz zu wenig da. Wer daher keinen Platz findet, muss als nächstes im Kreis stehen und den Schwarm neu mischen. Der Spieler in der Mitte kann auch „Schwarm“ sagen, wobei alle Mitspieler die Plätze wechseln müssen.

Im Anschluss kann im Kreis der Satz "Es war einmal..." durch die verschiedensten Plots ersetzt werden, was bereits der Arbeit am Subtext entspricht, denn der Text wird phantasievoll ergänzt. Der Satzanfang könnte mit „eine Wunderblume" erweitert werden, um daraus beispielsweise eine Übung zu Relativsätzen nach folgendem Schema zu gestalten: „Es war einmal eine Wunderblume, die mich klein (groß, stark, müde, blau, verrückt etc.) macht“. Diese könnten wiederum körperlich dargestellt werden. Sagt ein(e) Teilnehmer(in) zum Beispiel den Satz: „Es war einmal eine Wunderblume, die mich klein macht", tritt er oder sie dabei in den Kreis und findet eine passende Geste zum klein werden (natürlich kann hier auch der Komparativ geübt werden). In einem zweiten Schritt wiederholt der ganze Kreis gleichzeitig die vorgeführte Geste.

Zu Beginn der Kontextualisierungsphase steht eine 'Märchenstunde', in der die Lehrperson das gesamte Märchen vorliest. Entscheidet man sich, Illustrationen zu dem Text heranzuziehen, so wird damit eine unter Umständen notwendige Verstehenshilfe gegeben, aber die Phantasie der Teilnehmer auch stark gelenkt. Die einzelnen Abschnitte des Märchens werden an Gruppen verteilt, deren Mitgliederanzahl sich nach den im gegebenen Abschnitt 'auftretenden' Figuren richtet. Für den hier zitierten ersten Abschnitt wären es drei Teilnehmer pro Gruppe.

Es war einmal ein Schloss mitten in einem großen Wald. In dem Schloss wohnte eine Frau ganz allein, und das war eine Zauberin. Wenn jemand zu nahe an ihr Schloss herankam, so musste er stille stehen und konnte sich nicht von der Stelle bewegen, bis sie ihn wieder erlöste. Wenn sich ein Mädchen im Walde verirrt hatte, verzauberte die Zauberin es in einen Vogel, und den Vogel sperrte sie in einen Korb und den Korb trug sie in ihr Schloss. [...] Sie verzauberte sogar sich selbst. Am Tag machte sie sich zur Katze oder zur Eule, aber wenn die Sonne unterging, verwandelte sie sich wieder in einen Menschen. (Grimm, Steiner 2001) 
Schritt A: Details zur allgemeinen Situation herausfinden bzw. erfinden —Ein möglicher Subtext zu diesem Abschnitt in relativ einfacher Sprache, stichpunktartig verfasst, könnte sein:

Wann? Vor 200 Jahren wohnte die Zauberin in einem Schloss. Geschlossen. Keine Autos, keine Telefone, keine Fernseher. Leise. Langweilig. Wo? Das Schloss war tief in einem dunklen Wald. Wenige Wege. Wild. Kalt. Viele Steine, Pfützen und Äste auf dem Weg. Au! Viele sind verschwunden. Keiner weiß, wohin. Angst. Wer? Die Zauberin ist eine alte, einsame Frau. Hässlich. Dick. Allein. Sie spricht nur mit sich selbst. Sie putzt. Sie wartet. Aber wenn jemand kommt, wird sie wütend. Am Morgen kommt ein junger Mann. Er ist Student. Er hat seine Eltern besucht. Heute will er zurück zu seiner Universität. Er muss durch den großen Wald. Am Nachmittag verirrt sich ein Mädchen in den Wald. Klein. Allein.

Schritt B: Innere Monologe zu den einzelnen Figuren verfassen - Die Aufgabe, innere Monologe zu verfassen, kann sowohl der Gruppe als auch den einzelnen Teilnehmern überlassen werden. Es ist günstig, diese zunächst in mündlicher Form aus bestimmten Körperhaltungen heraus zu entwickeln, um sie anschließend schriftlich festzuhalten.

Beispiele:

Innerer Monolog der Zauberin: „Ich stehe am Fenster und schaue hinaus. Hohe Bäume. Dunkel. Gefängnis. Ich bin allein. Ich will anders sein. Es ist mein Wald. Macht. Ich liebe Macht! Ich will niemanden in meinem Wald. Ich bin allein. Wenn jemand kommt, verzaubere ich ihn. Ich mag Jungen nicht. Ich hasse Mädchen. Sie sind alle in Käfigen. Ich will sehen, wie sie leiden. Keiner ist stärker als ich. Ich bin allein. Ich verwandele mich heute in eine Katze. Miau!"

Innerer Monolog des Jungen: „Ich wandere zu meiner Universität. Ich war bei meinen Eltern. Mein Vater ist krank. Meine Mutter ist traurig. Ich habe Angst. Ich muss schnell durch den Wald. Aber ich will nicht. In Eile. Es ist dunkel. Viele sind im Wald verschwunden. Ich habe Angst. Oh, kann ich mich nicht mehr bewegen."

Innerer Monolog des Mädchens: „Ich will weg von zu Hause. Ich bin 12 Jahre alt. Meine Eltern lieben mich nicht. Fremd. Ich will weg. Egal wohin. Fremd. Ich gehe in den Wald. Es ist dunkel. Aber ich habe keine Angst. Ich will keine Angst haben. Ich will weg. Plötzlich bin ich in einem Käfig. Ich kann nicht mehr sprechen. Ich singe stumm."

Die Subtexte können zunächst in Bruchstücken von Sätzen assoziativ zusammengetragen, um in einem nächsten Schritt gemeinsam in vollständige Sätze gefasst zu werden. Die Wiederholung einzelner Schlüsselsätze hebt die wichtigsten Zustände hervor. Gleichzeitig kann die Ermutigung zu Wiederholungen auch zur dramatischen Verdichtung der szenischen Situation führen, anhand derer kurze szenische Übungen durchgeführt werden können, beispielsweise ein gemeinsames Experiment zu Körpersprache und Status auf der Grundlage des wiederholten Satzes der Zauberin „Ich bin einsam“ im Unterschied zur Wiederholung eines ihrer anderen Sätze wie „Ich liebe Macht!“ etc.. Das begrenzte 
Vokabular von Sprachlernern lenkt die Phantasie in bestimmte Richtungen, die nicht unbedingt der muttersprachlichen Interpretation entsprechen. Aber darin besteht gerade die Herausforderung: in der Beschränkung einen Raum der Freiheit und Kreativität zu entdecken.

Da in diesem ersten Abschnitt des Märchens sehr allgemeine Informationen über Ort, Zeit und Personen gegeben bzw. dazu erfunden werden, die für alle weiteren Abschnitte relevant sind, empfehle ich, diesen Abschnitt zunächst an die gesamte, in Untergruppen unterteilte Klasse zu verteilen, um in einer Zwischenauswertung die Subtexte und inneren Monologe zu vergleichen. Damit wird zugleich in die Arbeitsweise mit Subtexten und inneren Monologen eingeführt. An dieser Stelle kann bereits mit Improvisationen gearbeitet werden, in denen der Körperimpuls zum Zuge kommt. Die inneren Monologe können statt sprachlich auch körperlich präsentiert werden, zum Beispiel in Form einer Scharade: Im Falle des verzauberten Jungen führt ein Teilnehmer seine Figur in den gegebenen Umständen vor (durch den beängstigenden Wald eilend und schließlich erstarrend) und die Zuschauer tragen assoziierte Wörter (wie: Mann, jung, in Eile etc.) zusammen, um so gemeinsam zu einer neuen Ebene der Figurenbeschreibung zu gelangen. An die Auswertung der Subtexte zum ersten Abschnitt kann eine erste Einordnungsphase anschließen, die sich auf das Sprachmaterial aus den improvisierten Texten ergibt.

In der Intensierungsphase beginnt die Auseinandersetzung mit den Hauptfiguren des Märchens, die ich hier aus Platzgründen nicht an Textbeispielen erläutere. Der Text des gesamten Märchens wird abschnittsweise an verschiedene Gruppen verteilt, in denen Subtexte und innere Monologe erarbeitet werden. Wichtig ist, dass sich die Gruppen intern über die Ausgangssituation einigen, denn nur so können eindeutige Haltungen definiert werden. Dabei können die Subtexte in den Gruppen wiederum auch aus Improvisationen entstehen. Wenn es sich beispielsweise um die Szene handelt, in der Jorinde und Joringel sich im Wald verirrt haben und angstvoll Schutz beieinander suchen, so können die inneren Monologe aus der zusammengekauerten Körperhaltung heraus entwickelt werden und analog dazu Joringels Traum von der Wunderblume aus einer liegenden Position etc. Auch an diese Intensivierungsphase lässt sich gut eine Einordnungsphase anschließen, in der auf die sprachlichen Muster bzw. Probleme der vorgelegten Texte, die eventuell von den Teilnehmern in Form von individuellen Hausaufgaben weiter ausgearbeitet wurden, eingegangen wird.

Bleibt genügend Zeit für eine szenische Präsentation, so schlage ich vor, diese nicht auf den Ausgangstext des Märchens selbst zurückzuführen, sondern sie auf der Dramaturgie der jeweiligen Subtexte aufzubauen, was sicherlich zu einer abstrakteren Inszenierungsskizze führt, in der das vorher zusammen getragene Sprachmaterial noch einmal körperlich aktiviert und so vertieft werden kann. In diese Collage können alle Teilnehmer integriert werden, denn die Form der Collage lässt die Kombination der verschiedenen Subtexte und somit Sprünge in der Interpretation zu. In der Reflexionsphase kann wiederum stichpunktartig in der Fremdsprache (eventuell auch hier szenenweise dem Fragenkatalog folgend) die Dramaturgie der szenischen Collage zusammengetragen werden, 
wobei die individuellen Erfahrungen beim Spiel und im gesamten Prozess sicherlich besser in der Muttersprache auszutauschen sind, wenn es eine gemeinsame gibt.

\section{Gewinn}

Die Arbeit an Subtexten ist nicht reines Abfragen von Textverständnis, sondern kreatives Ergründen und Hinzufügen von Hintergrundinformationen zu einer Situation und/oder Figur. Es ist Textarchäologie, bei der in den unausgesprochenen Palimpsestes der Originaltexte jene Fundstücke ausgegraben werden, die aus der individuellen Perspektive des Lernenden ins Auge stechen.

In dem beschriebenen Ansatz wird nicht Einfühlung des Individuums gefordert, sondern eine Erfahrung der Gruppe geschaffen. Das bedeutet nicht, dass Emotionen keine Rolle spielen oder gar ausgeschaltet werden, aber die physische Aktion steht im Vordergrund der szenischen Handlung.

Die erfolgreiche Arbeit an Subtexten setzt folgende Haltungen bzw. Herangehensweisen voraus:

- partnerschaftliches Lehrer-Lerner- und Lerner-Lerner-Verhältnis

- Aktivität versus Passivität

- Entwicklung einer offenen Haltung zum Text

- Erproben von Haltungen 'am eigenen Leib'

- bewusstes Spiel

- präzise Fragen stellen, die der Situation und den Teilnehmern angepasst sind, dabei Antworten in ihrer Interpretationsbreite aufgreifen

- nach der kreativen 'Freilegung' der Subtexte: 'Entschlüsselung' der Fehler und Vertiefung anhand der von Lernern entwickelten Textbeispiele

Die Arbeit an Subtexten führt

- zur Erweiterung sowohl der sprachlichen als auch der theatralen Phantasie

- zu einer subjektiv-kreativen Textinterpretation

- zum Austesten von kulturellen wie persönlichen Grenzen

- zu mehr Selbstbewusstsein beim aktiven Anwenden der fremden Sprache

- zu kommunikativer Kompetenz 
Subtexte werden 'konstruiert' wie konstruktivistische Bilder, d.h. nicht nach psychologischen, sondern nach abstrakten Kriterien, was zur Erweiterung des Phantasiespielraums führt. Darüber hinaus kann der Konstruktivismus eines Meyerhold dem Sprachunterricht generell als Anregung dienen, dass Lernen ein kreativer, lehrerseits und lernerseits bewusster und aktiver Prozess ist, dessen Parameter immer wieder hinterfragt werden müssen.

Unter anderem durch die Arbeit an Subtexten trägt der Fremdsprachenunterricht zum "Prozess der Sprachbewusstwerdung" (Gnutzmann zitiert in Even 2003: 132) bei. Der hier zugrunde liegende Begriff des Textverständnisses beruht nicht auf der Haltung, dass alles abfragbar sei, sondern auf der Einsicht in die individuelle Interpretation des Textes. Wird der Lern- und Lehrvorgang als gemeinsame Inszenierung (cf. Schewe 1993) begriffen, kann ganz bewusst das 'Lernen des Lernens' gefördert werden. Und vor allem die Lust am Lernen, wie es Brecht formuliert hat:

Dieses Lernen sei lustvoll. Als eine Kunst / Werde das Lernen gelehrt, und auch das Behandeln der Dinge und Menschen / Lehret als Kunst, und Kunst auszuüben ist lustvoll. (Brecht 1966: 148)

\section{Bibliografie}

Ahrends, Günther (ed.) (1992): Konstantin Stanislawski. Neue Aspekte und Perspektiven. Tübingen: Schriftenreihe Forum Neues Theater

Bertolt Brecht (1966): Gedichte aus dem Messingkauf. In ders.: Über Theater. Leipzig: Reclam, 129-164

Brecht, Bertolt (1990): Theaterarbeit. Berlin: Henschel

Damásios, António (1994): Descartes' Error. Emotion, Reason and Human Brain. New York: Putnam Publishing

Eco, Umberto (1994): Lector in Fabula. München: Deutscher Taschenbuchverlag

Even, Susanne (2003): Drama Grammatik. Dramapädagogische Ansätze für den Grammatikunterricht Deutsch als Fremdsprache. München: iudicium

Goleman, Daniel (1995): Emotional Intelligence. New York: Bantam Books

Grimm, Jakob und Wilhelm; Steiner, Jörg (2001): Jorinde und Joringel. Hamburg: Carlsen

Heathcote, Dorothy; Bolton, Gavin (1998): Teaching Culture through Drama. In: Byram, Michael (ed.): Language Teaching in Intercultural Perspective.

Approaches through Drama and Ethnography. London, New York: Cambridge University Press, 160

Iser, Wolfgang (1984): Der Akt des Lesens. München: Fink

Lehmann, Hans-Thies (1999): Postdramatisches Theater. Frankfurt: Verlag der Autoren 
Meyerhold, Vsevolod (1967a): Der Schauspieler der Zukunft (1922). In:

Meyerhold, Tairov, Wachtangov: Theateroktober. Leipzig: Reclam, 101-104

Meyerhold, Vsevolod (1967b): Balagan (1912). In: Meyerhold, Tairov, Wachtangov: Theateroktober. Leipzig: Reclam, 64-100

Schewe, Manfred (1993): Fremdsprache inszenieren. Zur Fundierung einer dramapädagogischen Lehr- und Lernpraxis. Oldenburg: Zentrum für Pädagogische Praxis

Schmid, Herta (1992): Stanislavski und Meyerch'old. In: Ahrends, Günther (ed.) (1992): Konstantin Stanislawski. Neue Aspekte und Perspektiven. Tübingen: Schriftenreihe Forum Neues Theater, 65-84

Stanislawski, Konstantin (1986): Die Arbeit des Schauspielers an der Rolle. Berlin: Henschel

Vygotskij, Lev Semeonovic (2002): Denken und Sprechen. Psychologische Untersuchungen. Weinheim, Basel: Beltz Taschenbuch

Wagner, Betty Jane (1998): Educational Drama and Language Arts. What Research Shows. Portsmouth: Heinemann 